\title{
Phase II Study of Gemcitabine plus Cisplatin in Patients with Anthracycline- and Taxane- Pretreated Metastatic Breast Cancer
}

\author{
Jung Hwan Kim, M.D. ${ }^{1}$, Sung Yong Oh, M.D. ${ }^{1}$, Hyuk-Chan Kwon, M.D. ${ }^{1}$, Suee Lee, M.D. ${ }^{1}$, Sung-Hyun \\ Kim, M.D. ${ }^{1}$, Dae-Cheol Kim, M.D. ${ }^{2}$, Jin-Hwa Lee, M.D. ${ }^{3}$, Hyung-Sik Lee, M.D. ${ }^{4}$, Se-Heun Cho, M.D. ${ }^{5}$ \\ and Hyo-Jin Kim, M.D. ${ }^{1}$
}

Departments of ${ }^{1}$ Internal Medicine, ${ }^{2}$ Pathology, ${ }^{3}$ Diagnostic Radiology, ${ }^{4}$ Radiation Oncology and ${ }^{5}$ Surgery, Dong-A University College of Medicine, Busan, Korea

Purpose: Metastatic breast cancer patients are usually exposed to taxane and anthracycline as neoadjuvant, adjuvant and palliative chemotherapeutic agents. This study was designed to determine the efficacy and safety of the use of a gemcitabine and cisplatin (GP) combination treatment in patients with metastatic breast cancer that were pretreated with anthracycline and taxane.

Materials and Methods: We evaluated the use of a GP regimen $\left(1,000 \mathrm{mg} / \mathrm{m}^{2}\right.$ gemcitabine administered on days 1 and 8 plus $60 \mathrm{mg} / \mathrm{m}^{2}$ cisplatin administered on day $1 \mathrm{ev}$ ery 3 weeks) in 38 breast cancer patients who had received prior chemotherapy with anthracycline and taxane as an adjuvant or neoadjuvant therapy, or as a palliative therapy.

Results: The median patient age was 49 years (age range, $35 \sim 69$ years). The overall response rate was $28.9 \%$ in 11 patients (95\% confidence interval [Cl], $14 \sim$

\section{INTRODUCTION}

Breast cancer is a major cancer of women worldwide and the incidence of the disease has been increasing annually. For the past several years, breast cancer has been the most prevalent cancer among Korean women; the annual incidence may now exceed 10,000 cases (1). It has been estimated that metastatic breast cancer develops in $35 \sim 40 \%$ of all patients with breast cancer (2).

Metastatic breast cancer patients are usually exposed to anthracycline and taxane as neoadjuvant, adjuvant and palliative chemotherapeutic agents. Although anthracycline and the taxane are the most active first-line drugs for treatment, the cancers of many patients will progress and require the use of other chemotherapeutic agents (3). Among several agents that have

Correspondence: Hyo-Jin Kim, Department of Internal Medicine, Dong-A University College of Medicine, 3-1, Dongdaeshin-dong, Seo-gu, Busan 602-715, Korea. (Tel) 82-51-240-2951, (Fax) 82-51-2402951, (E-mail)kimhj@dau.ac.kr

Received May 16, 2008, Accepted August 27, 2008

This paper was supported by the Dong-A University Research Fund.
44\%). The median time to progression was 5.2 months (95\% Cl, $3.6 \sim 6.8$ months). Median survival was 19.5 months $(95 \% \mathrm{Cl}, 11.2 \sim 27.8$ months). Major grade $3 / 4$ hematological toxicity was due to leukopenia (36 of 157 cycles, $23.1 \%$ ). Non-hematological toxicity was rarely severe; grade $1 / 2$ nausea and vomiting were observed in $37.8 \%$ of the patients. There were no treatment related deaths.

Conclusions: Our results suggest that the use of gemcitabine plus cisplatin appears to be effective and has an acceptable toxicity profile in patients with advanced breast cancer that have been pretreated with anthracycline and taxane. (Cancer Res Treat. 2008;40:101-105)

Key Words: Breast neoplasms, Anthracycline, Taxane, Gemcitabine, Cisplatin

been used, gemcitabine and platinum compounds have been well-characterized single agents $(4,5)$, but there are few studies about the use of a combination of these two types of chemotherapeutic agents. Synergism between gemcitabine (a compound that inhibits DNA repair) and cisplatin (a compound that induces DNA damage) has been demonstrated in in vitro studies (6). Exposure to gemcitabine can counteract the cisplatin resistance that results from the up-regulation of DNA repair processes. Cisplatin enhances the rate of incorporation of gemcitabine, leading to apoptosis (7). Clinically, these agents have partially non-overlapping toxicity, as gemcitabine does not enhance cisplatin-induced nephrotoxicity or neurotoxicity, and cisplatin causes only mild myelotoxicity (8).

We have conducted this prospective phase II study in an effort to evaluate the efficacy and safety of the use of gemcitabine and cisplatin (GP) combination chemotherapy in patients with metastatic breast cancer that were pretreated with anthracycline and taxane.

\section{MATERIALS AND METHODS}

\section{1) Eligibility}

To be eligible for this study, patients were required to have a histologically confirmed diagnosis of carcinoma of the breast 
and at least one measurable lesion. This study included patients who had received anthracycline-based and taxane-based previous neoadjuvant, adjuvant and palliative chemotherapy regimens. Only patients with Eastern Cooperative Oncology Group (ECOG) performance status of grade $0 \sim 2$ were enrolled in this study. The patients had no active infections, no serious or uncontrolled concurrent medical illness and no previous history of other malignancies. Adequate hepatic, renal and bone marrow function was essential. The local ethics committee approved the study and informed consent was obtained from all patients before study entry.

\section{2) Treatment protocol}

Chemotherapy was administered through a chemoport placed in the subclavian vein or directly into a peripheral vein. The patients were administered $1,000 \mathrm{mg} / \mathrm{m}^{2}$ gemcitabine (1-hour infusion) on days 1 and 8 , and the patients were administered $60 \mathrm{mg} / \mathrm{m}^{2}$ cisplatin (over a 1-hour infusion) on day 1. Each cycle of chemotherapy was given every 3 weeks if the patient blood count had returned to an acceptable level (WBC: 3,000 $\times 10^{3} / \mu 1$; platelets: $70,000 \times 10^{3} / \mu 1$ ) and non-hematological toxic effects had resolved. The dosage of the subsequent cycles was adjusted according to the toxic effects that developed during the preceding cycle. If the hematological values were not reached by the date of the scheduled retreatment, therapy was delayed in weekly intervals, and if the hematological criteria were not fulfilled after a delay of two weeks, the patient was removed from the study. Doses of gemcitabine and cisplatin were reduced by $25 \%$ during subsequent cycles if the neutrophil and platelet count nadirs were $<500 \times 10^{3} / \mu 1$ or $<50,000 \times 10^{3} / \mu 1$, respectively, or if neutropenic fever developed. In addition, on chemotherapy day 8 , a complete bloodc count was performed before gemcitabine treatment. The administration of gemcitabine was omitted in cases of grade 4 hematologic toxicity on day 8 . The primary endpoint of this study was the tumor response rate and secondary endpoints included time to progression (TTP), overall survival and toxicities.

All patients received a standard supportive regimen consisting of hydration with normal saline for at least $3 \mathrm{~L} / 24$ hours, dexamethasone and the use of 5-HT3 inhibitors. Followup history and physical examinations, tumor measurements, and toxicity assessments were performed before each 3-week cycle of therapy. Toxicity was assessed using the National Cancer Institute-Common Toxicity Criteria (NCI-CTC), Version 3.0.

\section{3) Assessment of response}

A physical examination, complete blood counts, blood chemistry and chest $\mathrm{x}$-rays were obtained after each cycle. Response was assessed using WHO criteria (9). Computed tomography (CT) scans were repeated every three cycles or earlier in cases of clinical deterioration.

\section{4) Statistical methods}

This trial was designed to detect a response rate of $30 \%$ as compared to a minimal, clinically meaningful response rate of $10 \%$. A two-stage optimal design as proposed by Simon was adopted, with a statistical power of $80 \%$ to accept the hypothesis and 5\% significance to reject the hypothesis. Allowing for a follow-up loss rate of up to $20 \%$, the total sample size required was 35 patients with measurable disease. The time to progression (TTP) and overall survival (OS) were calculated from the initiation of treatment to the first observation of disease progression or death, respectively. All data were analyzed using SPSS software (version 12.0, Chicago-IL). Prognostic factors for OS, TTP and response rate (RR) were analyzed by use of Fisher's exact test, Kaplan-Meier and Cox regression analyses.

\section{RESULTS}

\section{1) Patient characteristics}

Between November 2002 and July 2007, 38 patients were

Table 1. Patient characteristics

\begin{tabular}{|c|c|c|}
\hline & No. of patients & $\%$ \\
\hline Total number of patients & 38 & \\
\hline \multicolumn{3}{|l|}{ Age } \\
\hline Median & 49 & \\
\hline Range & $35 \sim 69$ & \\
\hline \multicolumn{3}{|l|}{ Menopausal status } \\
\hline Premenopausal & 21 & 55.3 \\
\hline Postmenopausal & 17 & 44.7 \\
\hline \multicolumn{3}{|l|}{ PS $(\mathrm{ECOG})^{*}$} \\
\hline $0-1$ & 29 & 76.3 \\
\hline 2 & 9 & 23.7 \\
\hline \multicolumn{3}{|l|}{ Hemoglobin level } \\
\hline$\geq 12 \mathrm{~g} / \mathrm{dl}$ & 14 & 36.8 \\
\hline$<12 \mathrm{~g} / \mathrm{dl}$ & 24 & 63.2 \\
\hline \multicolumn{3}{|l|}{ Tumor marker (CA 15-3) } \\
\hline Normal & 25 & 65.8 \\
\hline Elevated & 13 & 34.2 \\
\hline \multicolumn{3}{|l|}{ Hormone receptor status } \\
\hline $\mathrm{ER}^{\dagger}$ and/or $\mathrm{PR}^{\ddagger}(+)$ & 22 & 57.9 \\
\hline $\mathrm{ER}^{\dagger}$ and $\mathrm{PR}^{\ddagger}(-)$ & 16 & 42.1 \\
\hline \multicolumn{3}{|l|}{$\mathrm{HER}^{\S} 2$ status } \\
\hline Negative & 27 & 71.1 \\
\hline Positive & 11 & 28.9 \\
\hline \multicolumn{3}{|c|}{ No. of prior palliative chemotherapy } \\
\hline 0 & 5 & 13.2 \\
\hline 1 & 8 & 21.1 \\
\hline$\geq 2$ & 25 & 65.7 \\
\hline \multicolumn{3}{|l|}{ No. of organs involved } \\
\hline 1 & 16 & 42.1 \\
\hline 2 & 12 & 31.6 \\
\hline$\geq 3$ & 10 & 26.3 \\
\hline \multicolumn{3}{|c|}{ Site of metastasis (multiple involved) } \\
\hline Lung & 21 & 55.3 \\
\hline Lymph nodes & 19 & 50.0 \\
\hline Liver & 10 & 26.3 \\
\hline Bone & 10 & 26.3 \\
\hline Skin and soft tissue & 9 & 23.7 \\
\hline
\end{tabular}

*Eastern Cooperative Oncology Group Performance Status, ${ }^{\dagger}$ estrogen receptor, ${ }^{\ddagger}$ progesterone receptor, ${ }^{\S}$ human epidermal growth factor receptor. 
assigned to be treated at the Department of Internal Medicine at Dong-A University Medical Center, Busan, South Korea. The characteristics of the 38 patients enrolled in this study are described in Table 1 . The median patient age was 49 years (age range, 35 69 years). More than half of the 38 patients (21 patients, 55.3\%) were premenopausal. The performance status (ECOG) was grade 0-1 in most of patients; nine patients $(23.7 \%)$ were grade 2 . In 24 patients $(63.2 \%)$, the hemoglobin level was $12 \mathrm{~g} / \mathrm{dl}$. The tumor marker CA15-3 was elevated in 13 patients $(34.2 \%)$. For the hormonal receptor status, expression of estrogen receptor (ER) and/or progesterone receptor (PR) was positive in $22(57.9 \%)$ patients; HER2 was positive in $11(28.9 \%)$ patients. A total of $33(86.8 \%)$ patients had received prior palliative chemotherapy of more than one regimen. For metastasis, there was one involved organ in five patients $(13.2 \%)$, two involved organs in 12 patients $(31.6 \%)$ and three or more involved organs in ten patients $(26.3 \%)$. The lung was the most common metastatic site (21 patients, 55.3\%), and lymph nodes (19 patients, 50.0\%), the liver (ten patients, $26.3 \%$ ), bone (ten patients, $26.3 \%$ ) and the skin and soft tissue

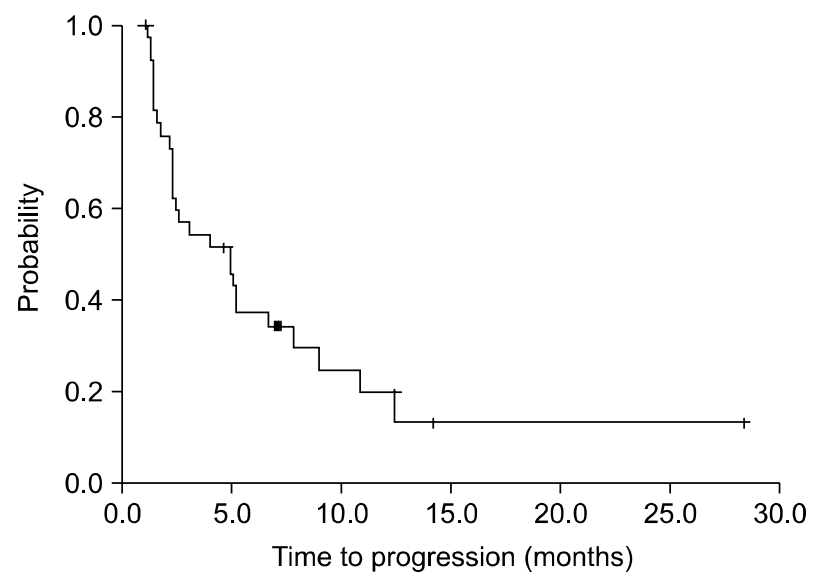

Fig. 1. A demonstration of the time to progression of the patients. The median TTP was 5.2 months (95\% CI; 3.6 6.8 months).

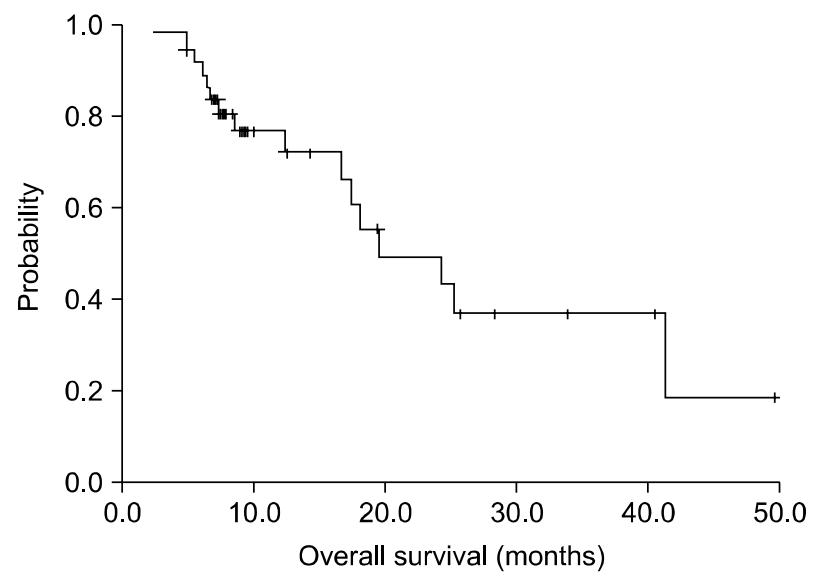

Fig. 2. The overall survival of patients. The median OS was 19.5 months (95\% CI; $11.2 \sim 27.8$ months). (nine patients, $23.7 \%$ ) were multiply involved.

\section{2) Tumor response and survival}

Of the 38 patients who were assessed for response, a partial response (PR) was seen in 11 patients $(28.9 \%$; $95 \%$ confidence interval $[\mathrm{CI}], 14 \sim 44 \%$ ), stable disease (SD) was seen in 14 patients $(36.8 \%)$ and progressive disase (PD) was seen in 13 patients (34.2\%). The median follow-up duration was 25.7 months. The median TTP was 5.2 months $(95 \% \mathrm{CI} ; 3.6 \sim 6.8$ months) (Fig. 1). The median OS was 19.5 months (95\% CI; $11.2 \sim 27.8$ months) (Fig. 2).

Only ECOG performance status 2 was a poor prognostic factor for TTP $(p=0.033)$. Other variations of patient characteristics were not significant prognostic factors for the response rate, TTP and OS (Table 2).

In case of disease progression with the use of gemcitabine and cisplatin chemotherapy, salvage chemotherapy was permitted and was performed in 22 of 25 patients with the use of various drugs. Trastuzumab was used in seven of 11 patients that were positive for ER2 expression. A response to salvage treatment was observed in eight patients (32\%).

\section{3) Dose administration and toxicity}

A total of 157 treatment cycles were administrated to the 38 patients with a median number of four cycles (range, $1 \sim 10$ cycles). The number of dose reduction cycles were $38(24 \%)$ among the 157 cycles. The most common reason for dose reduction was neutropenia $(74 \%)$, followed by thrombocytopenia (16\%) and hyperbilirubinemia (10\%). The relative dose intensities were $91 \%$ for gemcitabine and $93 \%$ for cisplatin.

An analysis of side effects showed that the toxicities were in general manageable and that anemia (grade 1 or 2 in $66.2 \%$ or 104 of 157 cycles) and neutropenia (grade 1 or 2 in $38.2 \%$ or 60 of 157 cycles) were the main toxicities. Grade 3 or 4 neutropenia were observed in $22.9 \%$ or 36 cycles, but only four episodes of non-fatal neutropenic fever were observed. Grade 1 or 2 vomiting was observed in 14 patients $(37.8 \%)$, and diarrhea (grade 1 in two patients), mucositis (grade 2 in two patients) and hyperbilirubinemia (grade1 or 3 in five patients) were reported. A grade 1 skin rash was observed in one patient. There were no treatment-related deaths (Table 3).

Table 2. Prognostic factors (p-value)

\begin{tabular}{|c|c|c|c|}
\hline & Response* $^{*}$ & $\operatorname{TTP}^{\dagger}$ & $\mathrm{OS}^{\dagger}$ \\
\hline Postmenopausal & 0.153 & 0.301 & 0.608 \\
\hline $\mathrm{ER}^{\ddagger}$ and/or $\mathrm{PR}^{\S}$ positive & 0.264 & 0.628 & 0.063 \\
\hline HER " positive & 0.088 & 0.399 & 0.999 \\
\hline Tumor marker increased & 0.571 & 0.637 & 0.897 \\
\hline Hemoglobin level $<12 \mathrm{~g} / \mathrm{dl}$ & 0.634 & 0.572 & 0.373 \\
\hline PS $(\mathrm{ECOG})^{\pi} 2$ & 0.179 & 0.033 & 0.114 \\
\hline Palliative chemotherapy history $(+)$ & 0.425 & 0.831 & 0.384 \\
\hline Organ involvement $\geq 2$ & 0.059 & 0.952 & 0.210 \\
\hline
\end{tabular}

*Fisher's exact test, ${ }^{\dagger}$ Kaplan-Meier and Cox Regression Analysis, ${ }^{\ddagger}$ estrogen receptor, ${ }^{\S}$ progesterone receptor, " human epidermal growth factor receptor, "Eastern Cooperative Oncology Group Performance Status. 
104 Cancer Res Treat. 2008;40(3)

Table 3. Toxicity of treatment

\begin{tabular}{|c|c|c|c|c|}
\hline & \multicolumn{4}{|c|}{ NCI-CTC* grade $(\%)$} \\
\hline & 1 & 2 & 3 & 4 \\
\hline \multicolumn{5}{|l|}{ Hematological $(n=157)^{\dagger}$} \\
\hline Anemia & $52(33.1)$ & $52(33.1)$ & $3(1.9)$ & \\
\hline Neutropenia & $36(22.9)$ & $24(15.3)$ & $10(6.4)$ & $26(16.7)$ \\
\hline Thrombocytopenia & $4(2.5)$ & $7(4.5)$ & $3(1.9)$ & \\
\hline Febrile neutropenia & & & & $4(2.5)$ \\
\hline \multicolumn{5}{|c|}{ Non-hematological $(\mathrm{n}=38)^{\ddagger}$} \\
\hline Vomiting & $11(28.9)$ & $3(8.9)$ & & \\
\hline Diarrhea & $2(5.3)$ & & & \\
\hline Mucositis & & $2(5.3)$ & & \\
\hline Hyperbilirubinemia & $4(10.5)$ & & $1(2.6)$ & \\
\hline Skin rash & $1(2.6)$ & & & \\
\hline
\end{tabular}

*National Cancer Institute-Common Toxicity Criteria, Version $3.0,{ }^{\dagger}$ per cycle, ${ }^{\dagger}$ per person; maximum toxicity of each patient.

\section{DISCUSSION}

Anthracycline and taxane usually have been used for metastatic breast cancer as a first or second line treatment, but many patients have disease progression and require the use of other chemotherapeutic agents. Vinorelbine, gemcitabine, cisplatin and capecitabine in single or combination regimens have been used, but there are no reported significant differences in the RR, TTP and OS.

Gemcitabine has been used as a single agent in the treatment of metastatic breast cancer (10-13). In a first-line setting, the response rates were $14 \sim 37 \%(10,13)$. When conducted in primarily pretreated patients, reproducible response rates were $17 \sim 29 \%$ (11,12). When a platinum compound was used as a single agent for metastatic breast cancer, the response rates were $32 \sim 50 \%$ in first-line therapy and approximately $10 \%$ in a salvage setting (14).

In this study, an RR of $28.9 \%$, a median survival of 19.5 months and a median TTP of 5.2 months were achieved. Previously reported treatment response rates of gemcitabinecisplatin combination chemotherapy have been variable, ranging from $26 \sim 80 \%(2,8,15,16)$. For patients with metastatic breast cancer pretreated with anthracycline and taxane, a response rate of $34.3 \%$, a median OS of 13.5 months and a TTP of 6.0 months have been reported (15). As compared with the findings in the present study, a slightly higher RR and TTP have been reported, and these findings may be due to a lower proportion $(50 \%)$ of the use of greater than third-line salvage chemotherapy than in our study (68.4\%). In addition, a lower proportion of patients with a positive HER 2 status (22\% versus $28.9 \%$ ) and a higher proportion of patients with a positive ER and/or PR status (95\% versus $57.9 \%$ ) may be contributing to a higher RR and TTP than in the present study.

For the use of other palliative chemotherapy regimens, the response rates that were observed include the following. For the use of gemcitabine-vinorelbine, an RR of $22 \sim 55.5 \%$ and a
TTP of $6.8 \sim 9.5$ months for first-line and second line treatments have been reported (17-19). For the use of capecitabine, an RR of $28 \%$ and a TTP of 4.9 months for second-line to fifth-line treatment have been reported (20). For the use of gemcitabinedocetaxel, an RR of $36 \sim 79 \%$ and a TTP of $7 \sim 8$ months for first-line to third-line treatment have been reported (21-23). For the use of gemcitabine-paclitaxel, an RR of $55 \sim 66.7 \%$ and a TTP of 11 months for first-line to third-line treatment have been reported $(24,25)$. For the use of gemcitabine-paclitaxel-trastuzumab, an RR of $52.5 \%$ and a TTP of 13.7 months for first-line treatment for HER2-positve cases have been reported (20).

A reason for a lower response rate in our study might be that $68.4 \%$ (26 patients) of all of the patients had undergone a GP regimen as more than a third-line salvage treatment. Although the response rate was lower than reported in previous studies, the TTP was comparable with the results of previous studies. A higher overall survival (19.5 months) may be due to the use of salvage chemotherapy. The RR of treatment after progression was also similar for the GP regimen. Follow-up was performed for a short duration in many patients (less than six months for 13 patients; 34\%), and data that was censored data was increased for OS and TTP (Fig. 1, 2).

Neutropenia, anemia, nausea, and vomiting were the main toxicities, the same findings as reported in a previous study. Most of these toxicities were Grade 1 or 2, and were usally manageable.

For the prognostic factors, positive expression of HER-2 was not a significant prognostic factor for survival. This may be due to the use of trastuzumab in only seven patients $(7 / 11,63.6 \%)$ of the positive HER-2 patients. Only performance status was a significant prognostic factor for TTP.

Most of the chemotherapy studies of metastatic breast cancer are phase II studies, and the benefit and harm of these therapies have not yet been confirmed. In our study, performance status was the only notable prognostic factor. Therefore, prior to the initiation of chemotherapy, it is necessary to evaluate the performance status to achieve a benefit for the patients. 


\section{CONCLUSION}

Our results suggest that the use of gemcitabine plus cisplatin appears to be effective and has an acceptable toxicity profile in patients with advanced breast cancer that have been pretreated with anthracycline and taxane.

\section{REFERENCES}

1. Shin HR, Jung KW, Won YJ, Park JG. 2002 annual report of the Korea Central Cancer Registry: based on registered data from 139 hospitals. Cancer Res Treat. 2004;36:103-14.

2. Wirk B, Perez E. Role of gemcitabine in breast cancer management: an update. Semin Oncol. 2006;33:S6-14.

3. Hortobagyi GN. Treatment of breast cancer. N Engl J Med. 1998;339:974-84

4. Seidman AD. Monotherapy options in the management of metastatic breast cancer. Semin Oncol. 2003;30(2 Suppl 3): 6-10.

5. Heinemann V. Role of gemcitabine in the treatment of advanced and metastatic breast cancer. Oncology. 2003;64:191206.

6. van Moorsel CJ, Veerman G, Bergman AM, Guechev A, Vermorken JB, Postmus PE, et al. Combination chemotherapy studies with gemcitabine. Semin Oncol. 1997;24:S7-17-S7-23.

7. Achanta G, Pelicano H, Feng L, Plunkett W, Huang P. Interaction of p53 and DNA-PK in response to nucleoside analogues: potential role as a sensor complex for DNA damage. Cancer Res. 2001;61:8723-9.

8. Nagourney RA, Link JS, Blitzer JB, Forsthoff C, Evans SS. Gemcitabine plus cisplatin repeating doublet therapy in previously treated, relapsed breast cancer patients. J Clin Oncol. 2000;18:2245-9.

9. Miller AB, Hoogstraten B, Staquet M, Winkler A. Reporting results of cancer treatment. Cancer. 1981;47:207-14.

10. Blackstein M, Vogel CL, Ambinder R, Cowan J, Iglesias J, Melemed A. Gemcitabine as first-line therapy in patients with metastatic breast cancer: a phase II trial. Oncology. 2002; 62:2-8.

11. Brodowicz T, Kostler WJ, Moslinger R, Tomek S, Vaclavik I, Herscovici V, et al. Single-agent gemcitabine as second- and third-line treatment in metastatic breast cancer. Breast. 2000;9: $338-42$.

12. Spielmann M, Llombart-Cussac A, Kalla S, Espie M, Namer M, Ferrero JM, et al. Single-agent gemcitabine is active in previously treated metastatic breast cancer. Oncology. 2001;60: 303-7.

13. Possinger K, Kaufmann M, Coleman R, Stuart NS, Helsing M,
Ohnmacht U, et al. Phase II study of gemcitabine as first-line chemotherapy in patients with advanced or metastatic breast cancer. Anticancer Drugs. 1999;10:155-62.

14. Martin M. Platinum compounds in the treatment of advanced breast cancer. Clin Breast Cancer. 2001;2:190-208.

15. Heinemann V, Stemmler HJ, Wohlrab A, Bosse D, Losem C, Kahlert $S$, et al. High efficacy of gemcitabine and cisplatin in patients with predominantly anthracycline- and taxane-pretreated metastatic breast cancer. Cancer Chemother Pharmacol. 2006; 57:640-6.

16. Smith IE. Overview of gemcitabine activity in advanced breast cancer. Semin Oncol. 2006;33(3 Suppl 9):S19-23.

17. Valenza R, Leonardi V, Gebbia V, Agostara B. Gemcitabine and vinorelbine in pretreated advanced breast cancer: a pilot study. Ann Oncol. 2000;11:495-6.

18. Stathopoulos GP, Rigatos SK, Pergantas N, Tsavdarides D, Athanasiadis I, Malamos NA, et al. Phase II trial of biweekly administration of vinorelbine and gemcitabine in pretreated advanced breast cancer. J Clin Oncol. 2002;20:37-41.

19. Nicolaides C, Dimopoulos MA, Samantas E, Bafaloukos D, Kalofonos C, Fountzilas G, et al. Gemcitabine and vinorelbine as second-line treatment in patients with metastatic breast cancer progressing after first-line taxane-based chemotherapy: a phase II study conducted by the Hellenic Cooperative Oncology Group. Ann Oncol. 2000;11:873-5.

20. Fountzilas G, Christodoulou C, Tsavdaridis D, KalogeraFountzila A, Aravantinos G, Razis E, et al. Paclitaxel and gemcitabine, as first-line chemotherapy, combined with trastuzumab in patients with advanced breast cancer: a phase II study conducted by the Hellenic Cooperative Oncology Group (HeCOG). Cancer Invest. 2004;22:655-62.

21. Fountzilas G, Nicolaides C, Bafaloukos D, Kalogera-Fountzila A, Kalofonos H, Samelis G, et al. Docetaxel and gemcitabine in anthracycline-resistant advanced breast cancer: a Hellenic Cooperative Oncology Group Phase II study. Cancer Invest. 2000;18:503-9.

22. Laufman LR, Spiridonidis CH, Pritchard J, Roach R, Zangmeister J, Larrimer N, et al. Monthly docetaxel and weekly gemcitabine in metastatic breast cancer: a phase II trial. Ann Oncol. 2001;12:1259-64.

23. Alexopoulos A, Tryfonopoulos D, Karamouzis MV, Gerasimidis G, Karydas I, Kandilis K, et al. Evidence for in vivo synergism between docetaxel and gemcitabine in patients with metastatic breast cancer. Ann Oncol. 2004;15:95-9.

24. Murad AM. Paclitaxel and gemcitabine as salvage treatment in metastatic breast cancer. Oncology (Williston Park). 2003;17(12 Suppl 14):26-32.

25. Delfino C, Caccia G, Gonzales LR, Mickiewicz E, Rodger J, Balbiani L, et al. Gemcitabine plus paclitaxel as first-line chemotherapy for patients with advanced breast cancer. Oncology. 2004;66:18-23. 